CLINICAL @LERT

\title{
Does chemoradiotherapy after intended curative surgery increase survival of gastric cancer patients?
}

\author{
A Cuschieri
}

Chemoradiotherapy after intended curative surgery for gastric cancer prolonged survival in patients with an adenocarcinoma of the stomach or gastro-oesophageal junction

T he randomised controlled trial reported by MacDonald et al documents an impressive increase in overall survival with postoperative chemoradiotherapy compared with surgical resection of gastric cancer alone (36 months $v 27$ months). Of equal importance is the finding of a significant reduction in the hazard ratio for relapse $(1.52$, 95\% CI 1.23-1.86; $\mathrm{p}<0.001)$. Although not the first randomised controlled trial to document a survival benefit from postoperative adjuvant therapy, ${ }^{1}$ it is the first to demonstrate a substantial survival advantage due to reduction of locally recurrent disease from postoperative adjuvant orthodox chemoradiation (425 $\mathrm{mg}$ fluorouracil $/ \mathrm{m}^{2}$ plus $20 \mathrm{mg}$ leucovorin $/ \mathrm{m}^{2}$ for five days, followed by 45000 cGy of radiation over a five week period) and brings hope to a depressing cancer with an unfavourable prognosis unless diagnosed at the Tl stage. Thus this North American randomised controlled trial merits close scrutiny.

The trial has adequate power and follow up, and analysis of the data seems appropriate. However, concerns have to be raised with respect to the nonstandardised nature of the surgical resections in the randomised patients. The only surgery related requirements for eligibility to this multicentre study stipulated by the protocol were: (i) resection with curative intent; and (ii) en bloc resection of tumour with negative margins. The account carries no definition of what constituted "resection with curative intent" for the purpose of the study. It appears that patients were identified (and hence recruited into the trial) on completion of a form by the surgeons defining the extent of lymphadenectomy. Thus there could not have been any serious attempt at standardisation/quality control of the resection. Consequently, there is a distinct possibility that this lack of standardisation of the surgical resection may have influenced the results in a spurious fashion, especially if the patients in the two arms had disease at different stages of advancement (pTNM). The characteristics of the patients in the two arms (see

Macdonald JS, Smalley SR, Benedetti J, et al. Chemoradiotherapy after

surgery compared with surgery alone for adenocarcinoma of the stomach or gastroesophageal junction. N Engl J Med 2001;345:725-30.

Question: Does chemoradiotherapy after surgery increase the survival of gastric cancer patients having an intended curative resection?

Design: Randomised controlled trial in nine US cancer centres.

Patients: A total of 556 patients with an adenocarcinoma of the stomach (mostly distal) or gastro-oesophageal junction (20\%), of which two thirds had T3 or T4 tumours and $85 \%$ had nodal metastases in resected specimens.

Intervention: Chemoradiotherapy started approximately one month after surgery. Chemotherapy, given before and after radiotherapy, consisted of fluorouracil and leucovorin, and radiotherapy of 4500 cGy at 180 cGy per day for five days per week for five weeks, given to the tumour bed and resection margins and to the regional nodes.

Outcome measures: The primary outcome was survival time and the secondary outcome was relapse free survival.

Results: Of 556 patients, 275 were randomised to surgery alone and 281 to surgery plus chemotherapy, of whom 181 (64\%) completed treatment as planned; $8 \%$ declined treatment, $17 \%$ stopped because of toxicity, and $10 \%$ discontinued treatment for other reasons, including disease progression. Most patients (54\%) had a partial resection of affected nodes (D0), 36\% had full resection of affected nodes (D1), and only 10\% had a D2 resection. Significant (grade 3 ) toxic effects were frequent and were haematological in $148(54 \%)$ and gastrointestinal in 89 (33\%). Other toxicity occurred in less than 10\% but three patients died of toxic effects attributed to chemoradiotherapy. After a median follow up of five years, median survival in the surgery only group was 27 months compared with 36 months in the chemoradiotherapy group (hazard ratio 1.35, 95\% confidence interval (CI) 1.1-1.7; $p=0.005)$. Median duration of relapse free survival was 19 months in the surgery only group compared with 30 months in the chemoradiotherapy group (hazard ratio for relapse 1.52, 95\% Cl 1.2-1.9; $\mathrm{p}<0.001$ ). Relapses were reported in $43 \%$ of the chemoradiotherapy group compared with $64 \%$ of the surgery only group.

Conclusion: Chemoradiotherapy given after intended curative surgery for gastric cancer prolonged survival by approximately one third.

table 1 in the article) appear to be similar although the T stage is not defined (clinical or pathological) and a most unusual index of node involvement (percentage involvement) is used for comparison, rather than the standard level of nodal involvement used in gastric cancer studies (N1, N2, N3). Hence, unless the authors clarify these issues, we cannot be certain that this randomised controlled trial compared patients with equivalent stages of the disease and that an R0 (no macroscopic evidence of residual disease) resection was undertaken always and in both groups. Even so, confirmation by other randomised controlled trials on adjuvant postoperative chemoirradiation is needed to reach the level III evidence that would mandate routine clinical usage.

Gut 2002;50:751

Author's affiliation

A Cuschieri, Department of Surgery and Molecular Oncology, Ninewells Hospital and Medical School, Dundee DDI 9SY, UK. a.cuschieri@dundee.ac.uk

\section{REFERENCES}

1 Neri B, de Leonardis V, Romano S, et al. Adjuvant chemotherapy after gastric resection in node-positive cancer patients: a multicentre randomised study. Br J Cance

1996;73:549-52. 